\title{
CORONARY ARTERY FISTULA, WHERE ARE WE NOW?"
}

\author{
Federico Benetti ${ }^{1}$, Jessica González ${ }^{1}$, and Gustavo Abuin ${ }^{2}$ \\ ${ }^{1}$ Affiliation not available \\ ${ }^{2}$ University of Buenos Aires Faculty of Medicine
}

October 1, 2021

\begin{abstract}
In the study "Long-term outcomes following surgical repair of coronary artery fistula in adults", Wada and associates, retrospectively evaluated 13 consecutive patients that underwent surgical repair of CAF No deaths, significant ST-T changes or CAF-related events were reported in a follow-up period of 66.2 months, and 1 patient showed poor contrast RCA\#2 on postoperative coronary $\mathrm{CT}$ with a myocardial scintigraphy showing no significant change compared to the preoperative state Coronary artery fistulas (CAF) are rare congenital or acquired malformations in the connection of the coronary vessels, first described by Krause in 1865 (2). They can be classified as coronary-cameral fistulas, which connect coronary arteries with any of the heart chambers, or coronary artery malformations, which connect coronary arteries with systemic or pulmonary vessels. Congenital CAFs are normally a result of abnormal embryological development, acquired CAFs are commonly a result of cardiac traumatic injuries, and iatrogenic CAFs are usually a result of interventional cardiac procedures. This condition is still highly undiagnosed, as around $75 \%$ of incidentally-found CAFs are small and clinically silent, but it is estimated that CAFs are present in about $0.9 \%$ of the general population In our experience, we have a CAF incidence of $0.05 \%$ in 10,000 cardiac surgeries, which have demonstrated beneficial outcomes of the surgical repair of CAFs in adults . Authors of this article must be congratulated for the successful development of the study and for the contributions to the literature on this rare condition
\end{abstract}

COMMENTARY: "TITULO? CORONARY ARTERY FISTULA, WHERE ARE WE NOW?" AUTHORS

Federico Benetti MD 1. Jessica Gonzalez MD 1. Gustavo Abuin MD 2

1. Benetti Foundation

Alem 1846 Rosario Argentina CP 2000

2. Universidad de Buenos Aires

Argentina

CORRESPONDIG AUTHOR:

Federico Benetti

Fundacion Benetti Alem 1846 Rosario Argentina CP 2000

FUNDING: NONE

CONFLICT OF INTEREST: NONE

KEY WORDS: Coronary artery fistula, coronary malformation, coronary surgery, coronary aneurysm

In the study "Long-term outcomes following surgical repair of coronary artery fistula in adults", Wada and associates, (1) retrospectively evaluated 13 consecutive patients that underwent surgical repair of CAF 
(coronary artery-pulmonary artery fistula, coronary artery-coronary sinus fistula, and both) at the Kokura Memorial Hospital between 2008-2019. The surgical procedures were performed under coronary artery bypass, and consisted of epicardial ligation of the fistula (46\%), direct closure of the fistula through a pulmonary artery incision (38\%), direct closure of the fistula through a coronary sinus incision (8\%), or the use of a patch closure of the fistula through coronary artery incision (8\%); patients who had comorbid cardiovascular disease underwent simultaneous fistula closure and surgery for their cardiac condition. The majority of CAFs originated from the right and left coronary arteries and drained into the main pulmonary artery, and preoperative findings included arrhythmias (31\%), low ejection fraction (30\%), and small regional ischemia (8\%). (1) No deaths, significant ST-T changes or CAF-related events were reported in a follow-up period of 66.2 months, and 1 patient showed poor contrast RCA\#2 on postoperative coronary CT with a myocardial scintigraphy showing no significant change compared to the preoperative state.

Coronary artery fistulas $(\mathrm{CAF})$ are rare congenital or acquired malformations in the connection of the coronary vessels, first described by Krause in 1865 (2). They can be classified as coronary-cameral fistulas, which connect coronary arteries with any of the heart chambers, or coronary artery malformations, which connect coronary arteries with systemic or pulmonary vessels. (3) Congenital CAFs are normally a result of abnormal embryological development, acquired CAFs are commonly a result of cardiac traumatic injuries, and iatrogenic CAFs are usually a result of interventional cardiac procedures. This condition is still highly undiagnosed, as around $75 \%$ of incidentally-found CAFs are small and clinically silent, (4) but it is estimated that CAFs are present in about $0.9 \%$ of the general population (5).

Although the vast majority of diagnosed CAFs are due to incidental findings during coronary catheterization procedures, a small number of patients develop symptoms of congestive heart failure, myocardial infarction (MI), or pulmonary hypertension secondary to the mechanism of "coronary steal phenomenon". The effects of high-pressure coronary arterial blood flow draining into a low-resistance venous circuit through the fistula bypasses smaller myocardium arterioles and capillaries and creates low-perfusion zones distal to the CAF, $(6,7)$ which, depending on the resistance (length, size and tortuosity) and site of the fistula connection (coronary vessel or cardiac chamber), can translate into dyspnea, angina, MI or volume overload symptoms. Chest X-ray and echocardiography may be helpful in the initial diagnosis and for uncovering any ensuing complications, further studies like multidetector computed tomography (CT) and magnetic resonance imaging (MRI) may be used to better delineate the fistula, while coronary catheterization and coronary angiography represent the gold standard for diagnosing CAF. (Dario)

According to American College of Cardiology and American Heart Association guidelines (8), surgical management is a class I recommendation for large CAFs regardless of the symptomatology and for symptomatic small to medium-size fistulas (including MI, arrhythmia, ventricular dysfunction of uncertain origin, and endocarditis). Treatment options include surgical ligation (may be done by epicardial or endocardial ligation) in large high-flow fistulas, tortuous fistulas, fistulas with multiple communications and drainage sites, presence of large aneurysms and if there is a need for simultaneous distal bypass grafting; and percutaneous transcatheter closure in fistulas with a proximal origin, single draining site, non-tortuous fistulas, fistulas with extra-anatomic terminations, patients without comorbid cardiovascular disease and old high-risk patients. (6)

In our experience, we have a CAF incidence of $0.05 \%$ in 10,000 cardiac surgeries, which have demonstrated beneficial outcomes of the surgical repair of CAFs in adults well as the beneficial effects of the concurrent intervention of CAFs during surgery of comorbid cardiac disease in preventing future complications related to CAFs. Authors of this article must be congratulated for the successful development of the study and for the contributions to the literature on this rare condition. Further studies, with larger sample size, should be done to evaluate the long term outcomes among the different procedures to treat CAF.

\section{REFERENCES}

1. Wada, Yuki, et al. "Long-term outcomes following surgical repair of coronary artery fistula in adults." Authorea Preprints (2021) 
2. Krause W. Ueber den Ursprung einer akzessorischen A.coronaria aus der A pulmonalis. Z Ratl Med 1865;24:225-9.

3. Rao, S. S., \& Agasthi, P. (2021). Coronary Artery Fistula. StatPearls, retrived from: https://wwwncbi-nlm-nih-gov.ezproxy.uniandes.edu.co:8443/books/NBK559191/ on September25, 2021

4. Challoumas D, Pericleous A, Dimitrakaki IA, Danelatos C, Dimitrakakis G. Coronary arteriovenous fistulae: a review. Int J Angiol 2014;23:1-10

5. Lim JJ, Jung JI, Lee BY, Lee HG. Prevalence and types of coronary artery fistulas detected with coronary CT angiography. AJR Am J Roentgenol. 2014 Sep;203(3):W237-43.

6. Yun, Gabin, Tae Hyun Nam, and Eun Ju Chun. "Coronary artery fistulas: pathophysiology, imaging findings, and management." Radiographics 38.3 (2018): 688-703.

7. Buccheri, Dario, et al. "Coronary artery fistulae: anatomy, diagnosis and management strategies." Heart, Lung and Circulation 27.8 (2018): 940-951

8. Warnes CA, Williams RG, Bashore TM, et al. ACC/AHA 2008 guidelines for the management of adults with congenital heart disease: executive summary - a report of the American College of Cardiology/American Heart Association Task Force on Practice Guidelines (Writing Committee to Develop Guidelines for the Management of Adults with Congenital Heart Disease). Circulation 2008;118(23):2395-2451. 